\title{
2 Exkurs: Zwischen Konkurrenz, Karriere und Kollaps
}

\author{
Andreas Hillert
}

\subsection{Männer ticken anders ...}

Der Auftrag, das vorliegende Kapitel zu schreiben, war eine Herausforderung, die nur wahre Männer bewältigen können: Risikoverhalten nach dem Motto: „Du hast keine Chance, nutze sie!“ Dass Männer anders ticken - als Frauen, an wen oder was sollte man sonst in diesem komparativen Kontext denken -, dass Männer also Konkurrenz-, Dominanz- und damit karriereorientierter sind, härter, konsequenter, risikobereiter, sich weniger sorgen und eher handeln ... (z.B. Bischof-Köhler 2010, 2011; Schulz et al. 2002) und es mit diesen Charakteristika schwer haben, all dies pfeifen die Spatzen ebenso von den Dächern dieser Welt, wie Barden die schwachen Seiten des starken Geschlechtes besingen. Männer sind so verletzlich und kriegen schließlich einen Herzinfarkt. Bei Männern werden Entzündungsprozesse stärker durch Stress stimuliert, Fibrinogen und C-reaktives Protein steigen (Toker et al. 2005). Männer trinken mehr und greifen schneller zu aufputschenden und konzentrationsfördernden Drogen (DAK-Gesundheitsreport 2009). Zudem hat Stress bei Männern Einfluss auf die Partnerinnenwahl, mit Händen im eiskalten Wasser fühlen sie sich eher nackten Frauen (auf Fotos) zugetan, die ihnen weniger ähnlich sind.

Ein solches filigran-melancholisches Gesamtbild einige Seiten lang anhand ausgewählter Daten, von niedersten Tieren bis zum Managerverhalten, zu bestätigen, wäre in hohem Maße erwartungskonform und entsprechend, zumal für Männer, wenig reizvoll. Einige Leser könnten dann auf Meta-Ebene ihre Wunden lecken, mit ihrem mickrigen Y-Chromosom Frieden schließen und müde konstatieren, dass die Wissenschaft in den letzten Jahrhunderten nicht viel weiter gekommen ist, als es Dichter und Denker schon immer waren. Richtig unangenehm würde es nur, wenn emanzipierte Frauen diese Seiten lesen würden. Implizit beinhalten die skizzierten Aussagen ja nichts anderes, als dass Frauen „von Natur aus“ eben anders anders sind. Selbst wenn man es noch so vorsichtig umschreibt, bliebe unter dem Strich übrig, dass Frauen weniger aggressiv und auf direktem Wege durchsetzungsfähig sind, dass sie sich 
eher im sozialen Netz verankern (und verstricken), letztlich viel besser zur familienfürsorglichen Brutpflege und weniger für das harte Berufsleben geeignet sind. Selbst Autorinnen, deren wissenschaftliche Untersuchungen eben solche Ergebnisse hatten, zeigten sich in der Diskussion ihre Resultate eifrig bemüht, diese schnell und heftig zu relativieren und sie damit soweit vom Tisch $\mathrm{zu}$ fegen, dass sie in keinem Fall irgendwie ethisch, sozialpolitisch oder sonstwie (miss-)verstanden werden können sollten und dürfen (Taylor et al. 200o). Wir alle sind fundamental von Emanzipation durchdrungen - und das ist gut so! Niemand soll diskriminiert werden, was allerdings Auswirkungen auf unsere Fähigkeit zu differenzieren haben könnte.

Um ideologischen Konflikten dieser Art zu entgehen, ließe sich andererseits konstatieren und ebenso substanziell mit Daten belegen, dass Männer und Frauen sich letztlich kaum unterscheiden, vom äußeren Erscheinungsbild und einigen männlichen Verhaltensmarotten abgesehen. Männer sind nichts Besonderes! Was dann aber die Berechtigung des vorliegenden, speziellen Buches zur Männergesundheit aus den Angeln heben würde. Man(n) bräuchte mit dem Schreiben gar nicht erst anzufangen. Das Dilemma ist offenkundig und fundamental: Biologische bis soziokulturelle, hormonelle wie politische Aspekte sind hinsichtlich der Frage nach dem üblichen Ticken und Verhalten der Männer (und Frauen) derart verwoben, dass objektive Bezugspunkte, um das Phänomen erfassen und werten zu können, de facto unvorstellbar sind. Und erst Recht keine Leser, die dem Thema neutral und unvoreingenommen gegenüberstehen. Es gibt nicht eine soziokulturelle Prägung männlicher und weiblicher Muster, die wir wie eine Fliege unterm Mikroskop sezieren könnten. Wir sind integraler Bestandteil unserer Muster: Wir sind nicht nur die Sezierenden, wir sind auch die Fliege! Aber wahre Männer resignieren nicht. Kapitulation ist für sie ein Fremdwort! Also, eine Chance haben wir nicht, aber schauen wir mal.

\subsection{Beruflicher und sozialer Stress im 21. Jahrhundert: Männer und Frauen in einem Boot}

Stress gab es immer. Stress, zumal in Form der oft thematisierten Reaktion auf einen sich anschleichenden Säbelzahntiger, ist langfristig ebenso überlebenswichtig, wie die Luft zum Atmen. Dass ungeachtet dessen gerade unsere nachindustrielle, postmoderne, sich als Informationszeitalter etikettierende Epoche Anspruch darauf erhebt, mehr als alle anderen durch das noch nicht allzulange so benannte Stress-Paradigma dominiert zu sein, ist argumentativ gut begründbar: Die fortschreitende technologische Entwicklung hat zu praktisch alle Lebensbereiche betreffenden Veränderungen geführt, gleichermaßen traditionelle Berufe und Sozialstrukturen ausgehebelt und durch Globalisierung neue Perspektiven eröffnet (Weber $u$. Hörmann 2007). Bei zunehmendem Tempo, Entgrenztheit und Unsicherheit fühlen sich allerdings immer weniger Menschen (zumal in den westlichen Industrienationen, die im globalen Ab- und Ausgleich relativ nicht gewinnen können) diesen Entwicklungen gewachsen, verunsichert, überfordert, also gestresst und ausgebrannt (Hillert u. Marwitz 2006). In einer groß angelegten Umfrage der EU gingen - aus gesundheitlichen Gründen - nur 65\% der Frauen und 73\% der Männer, die das 45. Lebensjahr erreicht und überschritten hatten, davon aus, ihre aktuelle berufliche Tätigkeit bis zum Alter von 6o Jahren ausüben zu können (Radoschewski 2009); nach den derzeit aus rententechnischen Gründen nötigen 65 bzw. 68 Jahren wurde nicht gefragt. 
Ob das Maß der derzeitigen Veränderungen tatsächlich das vorhergegangener Umbruchszeiten übersteigt, von diversen Völkerwanderwangen, der französischen Revolution bis zu den Weltkriegen, ist - da die davon unmittelbar Betroffenen nicht mehr leben - nicht wirklich bzw. vorzugsweise selbstgerecht diskutierbar. Ein wichtiger Unterschied $\mathrm{zu}$ vorangegangenen Umbruchszeiten liegt vielleicht darin, dass die Ausgangssituation diesmal eine vergleichsweise komfortable war. Nach diversen Wirtschaftswundern ging es den Allermeisten zumindest gut, die sozialen Absicherungen waren - bereits retrospektiv betrachtet - auf historischem Gipfel. Die Hoffnung auf immer weiteres exponentielles Wachstum war bereits vor der Bankenkrise naiv, heute hätte sie wahnhafte Qualitäten. Die realiter für viele damit verbundenen Grenzen, Kränkungen und Verluste sind möglicherweise schwerer verdaulich als es Frustrationen auf basalem Niveau gewesen wären.

Diese Entwicklungen haben natürlich für Menschen beiderlei Geschlechts fundamentale Implikationen. Konkurrenz und Karriere sind potenziell nach oben hin offen, Abstürze sind jederzeit möglich. Die Wahrscheinlichkeit eines Lebens auf langfristig stabiler, gesicherter und sozial etablierter Basis ist geringer geworden. Seinerzeit gab es zwischen Erfolg und Misserfolg eine breite Schicht von „Normalitäten“, die nun deutlich schmaler geworden ist. Die ehemals als aversiv erlebte, zu Rebellionen animierende Cewöhnlichkeit erscheint derzeit in romantischer Verklärung geradezu als Hort der Geborgenheit. Daten, mit denen sich die Realität und zumindest subjektive Relevanz dieses Stress-trächtigen Bildes für große Teile der Bevölkerung aufzeigen ließe, gibt es viele. Körperliche Schwerstarbeit müssen nur noch wenige leisten. Dafür wird die Erwerbsarbeit insgesamt zunehmend von quantitativen, qualitativen und vor allem psychosozialen Belastungen, sprich Stress, Zeitdruck, Multitasking, Entscheidungsdruck dominiert.

In diesem biopsychosozialen Kontext (Hillert et al. 2009) besonders prägnant ist der deutliche Anstieg von durch psychische und psychosomatische Störungen begründeten AU-Tagen (zwischen 1997-2004 waren es über 70\%). Bei insgesamt rückläufigen bzw. stagnierenden Krankheitstagen stiegen seit 2000 die durch Angststörungen (um 27\%) und Depressionen (um 42\%) begründeten Ausfalltage deutlich. Da sich die Menschheit in wenigen Jahren nicht relevant genetisch verändert haben kann, sind diese Zahlen letztlich nur im Kontext der skizzierten Entwicklungen interpretierbar ... (vgl. Linden u. Weidner 2005; DAK-Gesundheitsreport 2009; Bundespsychotherapeutenkammer 2010).

\subsection{Frauen haben es schwerer ...}

Die vorliegende Literatur zum Thema „Arbeit und Geschlecht“ ist in ihren Implikationen eindeutig: Die Benachteiligung von Frauen, praktisch in allen Erwerbsarbeit betreffenden Aspekten, scheint offenkundig (z.B. Habermann-Horstmeier 2007). Im Gegensatz zu traditionell als berufstätig verorteten Männern kommen Frauen, soweit sie sich nicht mit einem Leben als Hausfrau und Mutter bescheiden, um den Doppelbzw. Mehrfachbelastung bedeutenden Spagat zwischen Familie, Haushalt und Beruf nicht herum. Der damit verbundene Druck wurde, neben einer bei Frauen generell erhöhten Sensibilität und Bereitschaft, unangenehme Aspekte zu reflektieren, als eine mitentscheidende Ursache für die deutlich häufiger von Frauen erlebten somatischen/psychosomatischen und psychischen Beschwerden interpretiert. 
Eine konstruktive Auflösung des Rollenkonfliktes war und ist für viele Frauen schwierig: Wenn Mutterschaft und Familie angestrebt wird, bedeutet dies - zumal in Ländern ohne substanzielle Unterstützungsangebote für berufstätige Frauen - oft Abstriche oder Verzicht auf finanzielle Autonomie und berufliche Karriere. Wenn Emanzipation wörtlich nehmende Frauen primär ihre Karriere leben, geht dies umgekehrt oft mit Verzicht auf Mutterschaft und nicht selten auch auf Partnerschaft einher. Je höher das Bildungsniveau von Frauen, umso weniger Kinder haben sie. Übrigens: Je höher Frauen auf der beruflichen Karriereleiter steigen, umso mehr nehmen sie Männer-typische Verhaltensweisen an, einschließlich erhöhtem Alkoholkonsum (Hupfer 2007). Die zunehmende Diversifizierung des Rollenbildes „der erfolgreichen Frau“ respektive die erlebte (bzw. antizipierte) Unmöglichkeit gleichermaßen eine ideale Partnerschaft und Karriere zu verwirklichen, bedeutet de facto eine für viele Frauen nicht auflösbare Zwickmühle - der Anstieg u.a. von Essstörungen ist vor diesem Hintergrund sicher kein Zufall. Männer hingegen, zumal beruflich erfolgreiche, haben in der Regel eine (starke) Frau an ihrer Seite, Familie und damit ein institutionalisiertes, emotional stützendes Netzwerk. Darüber hinaus sind auch weiterhin viele Arbeitsbereiche, gerade auch in hinsichtlich Prestige und Geld attraktiven Leitungsfunktionen, von Männern dominiert. Ungeschriebene, nichtsdestoweniger eiserne Gesetze („gläserne Decken“), in denen Vorurteile, irrationale Ängste und rationale Aspekte ineinanderfließen, die das Vordringen von Frauen erschweren, wurden nachgewiesen.

\subsection{Männer aber auch ...}

Für unsere Väter muss es das Paradies auf Erden gewesen sein: Ihre patriarchalische Rolle war ungefährdet, in klar hierarchischen Männer-Gesellschaften wurde gearbeitet, Geld verdient und zu Hause wartete die umsorgende Gattin. Dass unsere Väter (ebenso wie unsere Mütter) kaum Möglichkeiten hatten, in gegebenem sozialen Kontext andere bzw. alternative Muster zu leben, unter der Last ihrer systemisch aufgebürdeten Rolle litten, scheitern und untergehen konnten, ist anzunehmen. Ungeachtet dessen waren eben diese „traditionellen“ Muster von Männlich- und Weiblichkeit ein Erfolgsmodell der Geschichte, ähnlich wie die Dinosaurier. Letztere sind bekanntermaßen längst ausgestorben, was von den „traditionellen“Verhaltensmustern der beiden Geschlechter (derzeit) noch nicht gesagt werden kann.

Diversen Erhebungen und tagtäglichen Erfahrungen u.a. von Therapeuten und Therapeutinnen zufolge, sind die etablierten männlichen Verhaltensmuster weiterhin in hohem Maße gegenwärtig. Männern fällt es demnach deutlich schwerer als Frauen, Gefühle bei sich wahrzunehmen, zu benennen und zu kommunizieren . Eine sensibel-selbstreflektierende Existenz, einschließlich der Fähigkeit, den eigenen Körper facettenreich zu spüren, gilt offenbar als für Gender-Männer wenig attraktiv. Vielmehr drängt es sie, über physiologische Grenzen hinweg zur Leistung. Konkurrenz wird gesucht und Balzverhalten gezeigt, auch wenn es um gar nichts geht.

Herbert Grönemeyer nannte sein berühmtes Lied von den starken schwachen Männern rückblickend eine „Realsatire“. Intellektuelle Distanz dieser Art ist auch ein Schritt zur Relativierung und damit Modifizierung der betreffenden Männlichkeitsmuster. Wie auch immer, in unterschiedlichen sozialen Gruppen dürften solche Pro- 
zesse unterschiedlich schnell und intensiv verlaufen (Keupp et al. 2008), in intellektuellen Kreisen vermutlich dynamischer als z.B. bei Spätaussiedlern aus den ehemaligen Ländern der UDSSR. Relativierung oder nicht, ein fundamental anderes Leitmotiv von Männer-Identität als eben die „traditionelle Prägung“ ist derzeit weder vorhanden noch am Horizont erkennbar. Entsprechend sind und bleiben sozial akzeptierte männliche Rollenmuster jenseits beruflichen Erfolges und starker Performance rar.

Dass Individuen im Gender-Konzept, wobei es um sozial determinierte Rollenmuster unabhängig vom biologischen Geschlecht geht, männliche wie weibliche Züge in interindividuell unterschiedlichem Maße vereinen können, macht die Sache zwar konzeptuell klarer, löst aber, zumindest für real existierende (biologische) Männer, das Problem im derzeitigen sozialen Kontext kaum. Erfolglose Männer bleiben Looser, erfolglose Frauen sind Opfer des Systems. Arbeitslose Frauen können sich, wohl oder übel, noch als Hausfrauen positionieren. Arbeitslose Männer, zumal in unteren sozialen Schichten, erst recht wenn sie sich als Hausmänner versuchen, laufen weiterhin Gefahr, als Witzfiguren wahrgenommen zu werden. Das klingt nicht nur hart, für Betroffene ist es de facto so. Entsprechend heftiger treffen die oben skizzierten globalen Entwicklungen die den traditionellen Rollenmustern verhafteten Männer (was wir somit mehr oder weniger alle sind). Angesichts dessen ist individuelle Verunsicherung, Überforderung und Bedrohung erheblich präsenter geworden. Traditionelles männliches, konkurrierendes und eskalierendes Rollenverhalten ist unter diesen Vorzeichen leider eine falsche, das Erleben von Ohnmacht provozierende Antwort.

\subsection{Stress-Response, methodische Grenzen und das Problem des unaufhaltsamen Wandels des untersuchten Gegenstandes}

Dass Männer und Frauen auf unmittelbare Bedrohung bzw. „Stress“, von der neuroendokrinologischen bis zur Verhaltensebene, zunächst ähnlich reagieren, ist seit dem Vater des Stress-Konzeptes, Hans Selye (bezogen auf unseren Kontext zusammenfassend z.B. Zapf u. Semmer 2004) und dem von Walter Cannon 1932 als „fightor-flight"-Muster beschriebenen Gesetz evident und angesichts der damit verbundenen evolutionären Vorteile gut begründet. Gleichwohl gelten Frauen im Umgang mit Stress-Belastungen traditionell als flexibler, ihre Stärke liegt mehr auf dem Gebiet der Beziehungsarbeit. Um das Überleben des Nachwuchses zu sichern, gelte es, wenn nötig, die eigene Sicherheit hinten anzustellen.

Ausgehend von solchen Überlegungen haben Shelly E. Taylor et al. vor nunmehr zehn Jahren die Konzepte und Befunde hinsichtlich geschlechtsspezifischer Stress-Response eingehend diskutiert (Taylor et al. 200o). Nachdem die Mehrzahl insbesondere der auf neuroendokrinologische Parameter fokussierenden Untersuchungen zum Thema, vor allem um Zyklus-bedingte Varianz auszuschließen, vorzugsweise männliche Wesen (meist Ratten) ins Visier genommen haben, war die Befundlage asymmetrisch. Gleichwohl fanden sich Hinweise darauf, dass Stress-Abläufe bei weiblichen Säugetieren durch niedrigere Spiegel männlicher Hormone (Androgene, insbesondere Testosteron) und höhere Oxytocin-Werte zu einem angemessener als ,tend-andbefriend“-(Fürsorge und Solidarisierungs-)Muster zu bezeichnenden Muster modifiziert werden. Weibliche Aggression verläuft demnach weniger offen bzw. Testoste- 
ron-gesteuert, gleichzeitig tendieren weibliche Wesen eher zu sozialer Nähe, was sich in Fürsorgeverhalten und dem Aufsuchen von Unterstützung (zumal unter Frauen) zeigt (und ähnlich durch exogene Oxytocin-Gabe stimulierbar ist). Mit den potenziell anti-emanzipatorisch missbrauchbaren Ergebnissen dieser Untersuchung konnten sich selbst deren Autorinnen nicht anfreunden. Entsprechend naheliegender war es, mit diesen Ergebnissen die zwischen den biologischen Geschlechtern unterschiedlichen Herzinfarkt-Raten (im prae-menopausalen Alter) zu erklären. Aktuelle Studien zeigen darüber hinaus, dass Stress bei Männern Entzündungsprozesse stimuliert, die ihrerseits den Weg zu arteriosklerotischen Prozessen, Hypertonus und letztlich Infarkten bahnen können (vgl. Toker et al. 2005).

\subsection{Unterscheiden sich die Stress-Bewältigungsstrategien von Lehrern und Lehrerinnen?}

Wie bereits erwähnt, sind insbesondere die psychosozialen Stress-Belastungen in den letzten Jahren im Arbeitsbereich deutlich gestiegen. Eine sozialem Stress besonders exponierte Gruppe sind Lehrerinnen und Lehrer. Die Frage, wie diese mit Stress umgehen, war Gegenstand einer Untersuchung, in der psychosomatisch erkrankte $(n=52)$ und gesunde Lehrer und Lehrerinnen $(n=93)$ miteinander verglichen werden sollten. Allen Teilnehmern wurden u.a. die fünf Kurzskalen des Stressbewältigungsfragebogens (vgl. Jahnke et al. 2002) vorgelegt, die

- Resignation („Neige ich dazu, zu resignieren?“),

- soziale Abkapselung („Gehe ich dem Kontakt mit anderen aus dem Weg?“),

- Entspannung („Tue ich etwas zu meiner Entspannung?“),

- Situationskontrolle (,Mache ich mir einen Plan, die Schwierigkeiten aus dem Weg zu räumen?"“) und

- Reaktionskontrolle („Versuche ich, Haltung zu bewahren?“) erfassen.

Mit einer selbstkonzipierten Skala wurde versucht, das Aufsuchen positiver Erlebnisinhalte („Versuche ich, mir etwas Gutes zu gönnen?“) abzubilden. Die klinischen Diagnosen der erkrankten Lehrer und Lehrerinnen wurden nach ICD-1o gestellt und u.a. emotionale Erschöpfung mit der entsprechenden Skala des Maslach-Burnout-Inventar (MBI) erfasst (Lehr et al. 2008).

Hinsichtlich möglicher Geschlechtsunterschiede hätten sich - ausgehend von traditionellen Rollenmodellen - diverse Hypothesen formulieren lassen, etwa, dass Lehrer weniger zur Resignation neigen sowie vor allem Situationskontrolle und in geringerem Maße Emotionskontrolle praktizieren als Lehrerinnen. Die Tatsache, dass Selbstbeschreibungs-Fragebögen per se Selbstkonzepte (und nur bedingt tatsächlich gezeigtes Verhalten) abbilden, hätte eher zur Überschätzung solcher Unterschiede führen müssen. Die empirischen Befunde entziehen all diesen Überlegungen den Boden: Die Stressbewältigungs-Profile von Lehrerinnen und Lehrern sind praktisch deckungsgleich (s. Abb. 1)!

Die primäre Fragestellung der Studie fokussierte auf mögliche Unterschiede zwischen gesunden und kranken Lehrpersonen. Diese Gruppen waren hinsichtlich soziodemografischer Parameter (Geschlecht, Alter, Partnerschaft etc.) parallelisiert. Zusammenhänge zwischen beruflichen Variablen (Berufserfahrung, Stundendeputat, Klassengröße, Schulgröße etc.) und dem Gesundheitszustand fanden sich nicht. Die 
Datensätze wurden dann einer hierarchischen Clusteranalyse unterworfen und die sich ergebenden Faktoren schrittweise auf Reliabilität und Validität geprüft. Als statistisch am stabilsten erwies sich eine Dreiclusterlösung (s. Abb. 2).

Cluster I-Vertreter zeigen ein flexibel-kompensierendes Bewältigungsmuster. Resignation und soziale Abkapselung sind hier gering, die Möglichkeiten sich zu entspannen und aktiv positives Erleben herbeizuführen werden genutzt. Gerade weil Belastungen vorhanden sind, wird das soziale Netz gepflegt und kann somit als Ressource in schwierigen Zeiten dienen. Vertreter dieses Musters verfügen sowohl über die Fähigkeit schwierige Situationen aushalten zu können, als auch über die Tendenz, solche Belastungen aktiv zu verändern. Spiegelbildlich dazu, im Sinne eines ruminativ-selbstisolierenden Verhaltens, ist das Cluster-III-Muster angelegt. Die Betreffenden erleben sich als resigniert, isoliert und wenn es darum geht, aktive Entspannung zu praktizieren oder positive Aspekte angesichts schwieriger Situationen zu finden, als weitgehend hilflos. Zwischen den beiden Extremen liegt ein als inkonsistent-kompensierende Bewältigung charakterisierbares Cluster II. Eine mäßig-resignative Grundhaltung korrespondiert hier mit der Fähigkeit, sich aktiv entlasten/entspannen zu können bei gleichzeitiger Tendenz, Belastungen passiv zu erdulden.

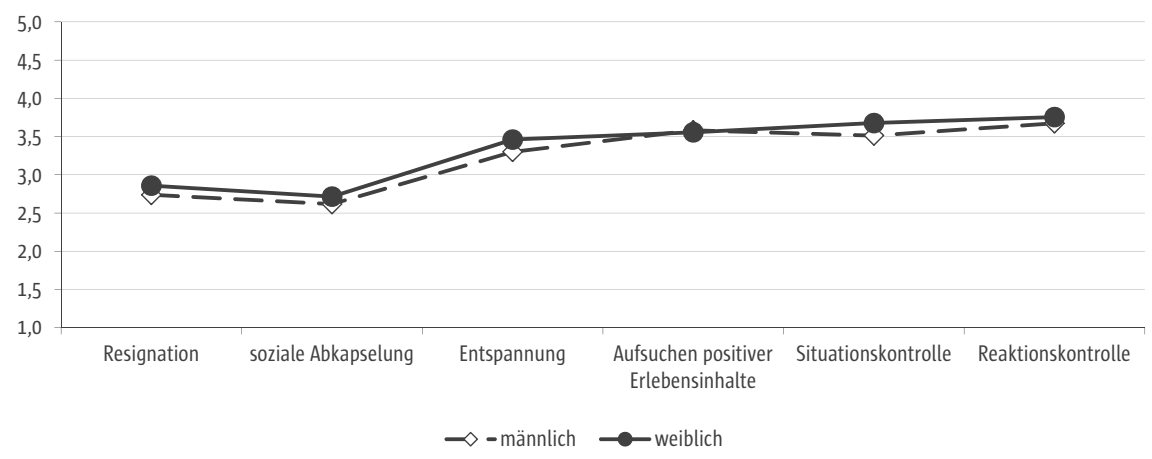

Abb. 1 Stressbewältigungsmuster psychosomatisch erkrankter und gesunder Lehrerinnen und Lehrer: geschlechtsbezogene Auswertung

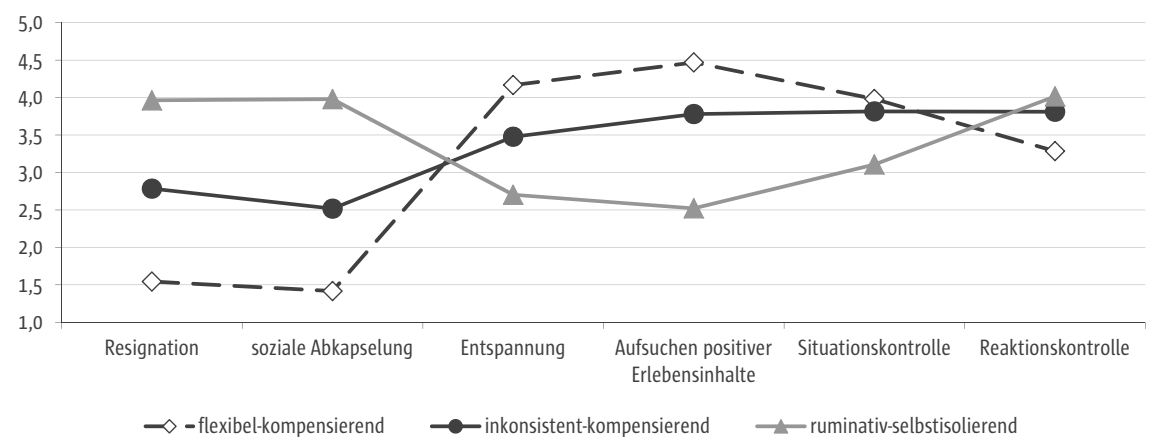

Abb. 2 Stressbewältigungsmuster psychosomatisch erkrankter und gesunder Lehrpersonen 
Beim Abgleich der jeweiligen Muster mit dem Gesundheitszustand - wobei es wie gesagt keine Geschlechtsunterschiede gab! - zeigte sich die hohe Relevanz des Ansatzes: $98 \%$ der Lehrpersonen mit flexibel-kompensierendem Bewältigungsmuster (Cluster I) und $76 \%$ der inkonsitent-kompensierende Bewältigung praktizierenden Stichprobe gehörten zur in der Schule aktiven, gesunden Gruppe. Von den angesichts beruflicher Belastungen mit ruminativ-selbstisolierendem Verhalten reagierenden Personen waren hingegen nur 21\% gesund, 79\% befanden sich in stationärer Behandlung! Parallel dazu zeichneten sich Custer-III Personen, relativ zu den Vertretern der beiden anderen Gruppen, durch signifikant höhere Depressivität, emotionale Erschöpfung, negative subjektive Erwerbsprognose, geringe Arbeitszufriedenheit und geringes Wohlbefinden bzw. Lebensqualität aus. Ticken Männer und Frauen, zumindest Lehrerinnen und Lehrer, demnach gleich, nur eben - geschlechtsunabhängig - einige besser und andere problemträchtiger (vgl. Lehr et al. 2008)? Personenmerkmale spielen hinsichtlich der Stressbewältigung unbestreitbar eine sehr große Rolle. Der relative Anteil von Sex- und/oder Gender-Aspekten im Rahmen dieser Persönlichkeitsmerkmale scheint aber, zumindest in bestimmten Berufsgruppen und bezogen auf berufliche Belastungen, gering zu sein.

\subsection{Burnout: Die Batterien sind leer, ich kann nicht mehr, Herr Doktor!}

Der Entdecker des Phänomens und gleichzeitig der erste Mensch, der sich als „ausgebrannt“ diagnostizierte, war ein Mann. Herbert Freudenberger (zusammenfassend Hillert u. Marwitz 2006), als Kind dem Holocaust entkommen, war zeitlebens in hohem Maße sozial engagiert und auf Sicherheit bedacht. Als Psychoanalytiker arbeitete er täglich zumindest 8 Stunden in eigener Praxis. Anschließend widmete er sich oft bis weit nach Mitternacht sozialen Aufgaben, z.B. in Einrichtungen für Mitglieder benachteiligter Randgruppen. Bei mehr als zwölf Arbeitsstunden/Tag wundert es auch ihn - im Nachhinein - nicht, dass seine Leistungsfähigkeit abnahm und er sich schließlich körperlich immer schlechter, müde, kraft- und lustlos fühlte. Häufig wurde er von Infekten, Verdauungsproblemen, Kopfschmerzen und Schlafstörungen geplagt. Im Kontakt zu Mitmenschen zeigte er sich reizbarer und unflexibel. Im Gegensatz zu vielen Menschen, die vor und nach ihm Ähnliches erlebten, behielt er soweit einen klaren Kopf, dass er seine Situation reflektieren, beschreiben und in einem kleinen, epochalen Aufsatz („stuf burnout“) publizieren konnte. Burnout betreffe in sozialen Berufen tätige Menschen, die mehr geben wollen als längerfristig Menschen-möglich sei. Diese Dynamik werde durch systemischen Druck, etwa die Forderungen von Vorgesetzten verstärkt. Eben - und nur - hieraus resultiere Burnout; mit einer seelischen Erkrankung, etwa Depressionen, habe das nichts zu tun. Verhindern und behandeln, systemisch wie individuell, lasse sich Burnout entsprechend nur durch einen angemessenen Umgang mit den eigenen Kraftreserven.

Die Psychologin Christina Maslach konzeptualisierte wenig später Burnout als ein Syndrom aus emotionaler Erschöpfung, Depersonalisierung und reduzierter persönlicher Leistungsfähigkeit. Das Phänomen sei Folge von Arbeitsüberlastung. Der von ihr entwickelte Fragebogen, das Maslach-Burnout-Inventar, ist das weltweit im Kontext Burnout-Messung am weitesten verbreitete Instrument. Wer auf die Frage „Ich fühle mich von meiner Arbeit ausgelaugt?“ mit zumindest „einige Male im Jahr“ 
antwortet, bekommt einen Punkt im MBI, „täglich“ würde mit 6 Punkten kodiert. Entgegen der ursprünglichen Idee von Herbert Freudenberger korrelieren MBI-Werte jeweils im mittleren Bereich mit fast allem, mit Stresserleben, Arbeits(un)zufriedenheit, Depressivität, Ängstlichkeit, emotionaler Labilität. Ein eigenständiges Phänomen ist (zumindest das mit dem MBI gemessene) Burnout nicht, sondern eine recht diffuse Gemengelage. Eine Interpretation all dieser Daten bleibt schwierig, schon deshalb, weil unklar bleibt, was die Fragebögen letztlich messen. Sie messen eben u.a. Depressivität. Dass Frauen von Depressionen häufiger betroffen bzw. diesbezüglich offener sind, ist bekannt ... (zusammenfassend Rohde u. Marneros 2007). Ungeachtet dessen waren (und sind) viele davon überzeugt, dass Frauen (Doppelbelastung!) besonders häufig von Burnout betroffen sein müssten. In einigen Untersuchungen mit dem MBI und anderen Fragebögen, etwa dem AVEM (Schaarschmidt u. Fischer 2001, 2003), bei umschriebenen Personengruppen, gab es solche Befunde, bei anderen allerdings auch nicht und wenn man die Vielzahl aller Erhebungen zusammenfasst, gab und gibt es zwischen den Geschlechtern bezüglich des BurnoutErlebens keine systematischen Unterschiede (Burisch 2010). Wenn, dann waren es wiederum eher besondere Konstellationen bzw. besondere Personengruppen, die aus dem Rahmen fielen, wobei Sex und Gender nur einer der die Besonderheit ausmachenden Faktoren war.

\subsection{Typisch weibliche versus männliche Stress-Reaktionen?}

Ein grundlegender Unterschied im Hinblick auf geschlechtsspezifische „Stress“-Abläufe wird in einer eher emotionalen, „expressiven“ Reaktion von Frauen und einem eher „instrumentellen“ Umgang bei Männern vermutet. Der „self-assertive Fragebogen“ (PAQ) (Runge et al. 1981; Sieverding et al. 2005) bildet in der deutschen Version jeweils auf fünf-Punkteskalen eben diese Aspekte ab:

v "Instrumentalität“ (Unabhängigkeit, Aktivität, Selbstvertrauen, Entscheidungsfreudigkeit, Durchhaltevermögen, Durchhaltevermögen auch unter Belastungen, Überlegenheitsgefühl) und

- „Expressivität“ (Freundlichkeit, Wahrnehmung der Gefühle anderer, Verständnis anderer, Fähigkeit sich unterordnen zu können, Warmherzigkeit u.a.).

In einer Studie von Monika Sieverding (Sieverding et al. 2005) wurden Studentinnen und Studenten einem Stress-Test unterzogen. Konkret ging es um ein improvisiertes Vorstellungsgespräch, dem sich die Probanden und Probandinnen zu stellen hatten. Dabei wurden sowohl die subjektive Stressbelastung als auch die objektiven Parameter (Puls und Blutdruck) erfasst. Es zeigte sich, dass Frauen auf die soziale Stresssituation insgesamt zwar emotionaler reagierten, sich also als „gestresster“ erlebten, ohne dass sich jedoch dafür auf physiologischer Ebene Unterschiede fanden. Darüber hinaus zeigte sich, dass Menschen, deren Geschlechtsrollenprofil ,instrumentell“ (und insoweit eben traditionellen männlichen Mustern ähnlich) akzentuiert war, unabhängig vom biologischen Geschlecht, subjektiv weniger stressbelastet waren aber gleichzeitig deutlich höhere körperliche Reaktionen zeigten. Demnach wäre für die über den mehr oder weniger emotionalen Moment hinausgehenden Stress-Folgen weniger das biologische sondern das soziale Geschlecht, also Gender, entscheidend. Risikofaktor ist demnach nicht das z.B. männliche Geschlecht, sondern ein 
die traditionell als männlich geltenden Charakterzüge für sich in Anspruch nehmendes Selbstkonzept.

Auch in dieser Studie waren die Ergebnisse zwar statistisch signifikant. Letztlich handelt es sich aber eher um Nuancen denn um kategorische Unterschiede. Zudem wurde deutlich, wie sensibel alle diese Studien sind, wenn die Studienbedingungen auch nur minimal modifiziert werden. Wie bzw. welche Art von Stress wird induziert? Sozialer Stress (freier Vortrag, Vorstellungsgespräch etc.) ist eine ganz andere Kategorie als schriftliche bzw. am Computer zu bearbeitende Leistungstests. Was wird gemessen? Subjektives Stress-Erleben und/oder physiologische Parameter? Wobei als Risikofaktor zumal im kardio-vaskulären Bereich dann weniger z.B. der akute Blutdruckanstieg sondern die Entspannungsfähigkeit danach (Recovery) wichtig wäre. Zudem ist damit zu rechnen, dass sich soziale und berufliche Gruppen deutlich hinsichtlich der Gender-Akzentuierungen unterscheiden. Darüber hinaus dürfte die Auswahl bzw. Selektion der Probanden und Probandinnen darüber entscheiden, ob bzw. auf welcher Ebene sich Geschlechts- und/oder Gender-Unterschiede finden. Die allermeisten experimentellen Studien zu menschlichem Stress-Verhalten wurden an Studentinnen und Studenten durchgeführt, also angehenden Akademikern, deren Sozialisation mehrheitlich traditionelles geschlechtsspezifisches Rollenverhalten in sozial geläutertem Maße beinhaltet haben dürfte. Eben dies gilt auch für die Lehrerinnen und Lehrer der zitierten Untersuchung und dürfte das bezüglich der Geschlechtsunterschiede negative Ergebnis erklären. Lehrer ist - von Ausnahmen abgesehen - kein Beruf, in den sich Macho-Männer oder aber zart-erotisierte Frauen drängen. Möglicherweise ließen sich Geschlechts- und Gender-Unterschiede der Stress-Response im Vergleich zwischen Deutschrussen ohne Schulabschluss und Kinderkrankenschwestern deutlicher aufzuzeigen? Solche Männer dürften dann tatsächlich ganz anders ticken als solche Frauen, wobei die Frage, inwieweit diese Unterschiede durch Geschlecht, Gender und/oder die sozialen Gruppen (vgl. Broding 2007) determiniert sind, spannend bzw. zur Quadratur des Kreises werden könnte. Und die üblicherweise $\mathrm{zu}$ fordernden Untersuchungen von für die Bevölkerung insgesamt repräsentativen Gruppen dürften jenseits des in solchen Studien Machbaren liegen. Jedoch ist Eile geboten! Es besteht die Gefahr, dass sich die aktuellen Unterschiede in den Gender-Profilen im Laufe der weiteren Entwicklungen und Emanzipationen nivellieren, sich inhaltlich anders konstellieren und damit in der derzeitigen Form schließlich (fast) ganz verschwinden.

\section{Ausblick}

Es ist ein bekanntes Phänomen - nicht nur, aber auch - wissenschaftlicher Annäherung, dass sich Konturen, je näher man ein Bild bzw. eine Frage ins Visier nimmt, verwischen. Und wenn dann noch bestimmte Aspekte des Bildes gesellschaftspolitisch bedenklich sind, macht dies die Diskussion heißer, das Ergebnis aber noch unbefriedigender.

Im Fall der Frage, ob Männer anders ticken, ist unser spontaner, unsere Erwartung bestimmender Ausgangspunkt eher weniger eine wissenschaftliche Hypothese, sondern die spontan, im täglichen Leben erfahrenen Geschlechtsunterschiede: Zwischen dem Marlboro-Mann, seiner zivilisierteren Bogner-Variante („höchste Ansprüche sind für ihn selbstverständlich“) und der reizend geschminkten Blondine mit dem kessen Minirock liegen augenscheinlich Welten. Entsprechend anders müssten doch diese Männer ticken! Marlboro und Minirock sind sicher prägnante Schemata, aber unsere Realität sieht meistens 
anders aus. Angesichts des uniformen Outfits aus Jeans und Pullover, in der sich die meisten Jugendlichen und weniger jugendlichen Menschen heute offenbar am wohlsten fühlen, wobei Geschlechtlichkeit auf vergleichsweise dezente Aspekte reduziert wird, dürfte sich die Erwartungshaltung an das jeweils andere Ticken hingegen automatisch relativieren. Ungeachtet der jeweiligen Moden sind und bleiben wir soweit in sexuelle Themen involviert, dass es aus der spannungsreichen Doppelrolle von sezierter Fliege und analysierendem Forscher kein Entkommen geben wird. Ob Auswege aus dieser Zwickmühle letztlich wünschenswert wären, möge jeder für sich entscheiden.

Zusammenfassend wird deutlich, dass es hinsichtlich des Erlebens und des Umganges mit Stress, so wie er zwischen Konkurrenz, Karriere und Kollaps ubiquitär ist, weniger um das biologische Geschlecht, sondern um "Gender", also das durch traditionelle Muster geprägte und individuell internalisierte soziale Geschlecht, gehen dürfte. Letzteres erklärt derzeit Unterschiede in Art und Ausmaß von Stress-Response besser. Dass die Gender-Forschung mit ihren aktuellen Paradigmen zu letztendlich gültigen Ergebnissen führen wird, ist jedoch unwahrscheinlich. Indem sich traditionellen Muster zwangsläufig weiter relativieren und sich damit unsere Umwelt und Sozialisation anders ausrichten wird, wird sich auch dieses Phänomen verschieben. Übrig bleiben Individuen mit mehr oder weniger hohen Testosteronspiegeln und Adrenalin-Anstiegen auf normierte Reize respektive mehr oder weniger instrumentellen, emotionalen, expressiven, kommunikativen, kontemplativen etc. Mustern im Umgang z.B. mit Stressoren unterschiedlicher Art. Der Trend zu größerer Individualität jenseits etablierter Muster ist schon jetzt unverkennbar. Dabei geht es nicht um Individualität im romantischen Sinn, um Selbstfindung nach den im klassischen Bildungsbürgertum formulierten Idealen oder um postmodern-narzisstische Individuen, wie sie in Form von Superstars und Super-Modellen gesucht werden, sondern um Menschen, die sich bei abnehmenden sozialen und materiellen Sicherheiten im Sinne einer Ich-AG behaupten müssen, die professionelles "networking" betreiben, sich mit „total dedication" zu etablieren versuchen und den Kern ihrer Individualität hinter einem auf die jeweilige Zielgruppe abgestimmten uniformen Auftreten bewusster kontrollieren als es bereits heute in bestimmten Branchen üblich sein mag. Auch wenn in einigen Jahrzehnten die uns selbstverständlichen Gender-Kategorien historische Relikte sein werden, die biologischen Geschlechtsunterschiede werden (vorerst) bleiben. Wie diese Unterschiede dann im sozialen Kontext gelebt und erlebt werden, bleibt abzuwarten. Ungeachtet dessen war und ist entscheidend, wie Sie - welchen biologischen Geschlechts und welchen Genders auch immer - mit Stress-Belastungen umgehen, welche Strategien (z.B. zwischen ruminativ-selbstisolierend und aktiv-copend) Ihnen zur Verfügung stehen. Zumindest diesbezüglich gibt es klare Evidenzen und entsprechend - wenn nötig - konkrete Handlungs- bzw. Behandlungsmöglichkeiten (z.B. Hillert et al. 2007).

\section{Literatur}

Bischof-Köhler D (2010) Evolutionäre Grundlagen geschlechtstypischen Verhaltens. In: Steins G (Hrsg.) Handbuch Psychologie und Geschlechterforschung. Wiesbaden: VS Verlag für Sozialwissenschaften, S. 153-172

Bischof-Köhler D (2011) Von Natur aus anders. Die Psychologie der Geschlechtsunterschiede. 4., überarbeitete und erweiterte Auflage. Stuttgart: Kohlhammer

Broding H Ch (2007) Working Poor. In: Weber A, Hörmann G (Hrsg.) „Mensch und Neue Arbeitswelt“ - Psychosoziale Gesundheit im Beruf. Stuttgart: Gentner Verlag, S. 352-366

Bundespsychotherapeutenkammer (Hrsg.) (2010) Komplexe Abhängigkeiten machen psychischkrank - BPtk-Studie zu psychischen Belastungen in der modernen Arbeitswelt, Pressekonferenz der Bundespsychotherapeutenkammer am 23. März 2010, www.bptk.de

Burisch M (2010) Das Burnout-Syndrom: Theorie der inneren Erschöpfung. Springer-Verlag, Berlin, 3. Aufl.

DAK (2009) DAK-Gesundheitsreport 2009, www.dak.de/content/filesopen/Gesundheitsreport_2009.pdf auch die Reporte anderer Krankenkassen sind im Internet verfügbar: www.bkk.de; www.barmer-gek.de; www.tkonline.de 
Egger A (2007) Arbeitslosigkeit. In: Weber A, Hörmann G (Hrsg.) „Mensch und Neue Arbeitswelt“ - Psychosoziale Gesundheit im Beruf. Stuttgart: Gentner Verlag, S. 375-389

Habermann-Horstmeier L (2007) Gender und Arbeitswelt. In: Weber A, Hörmann G (Hrsg.) „Mensch und Neue Arbeitswelt" - Psychosoziale Gesundheit im Beruf. Stuttgart: Gentner Verlag, S. 401-413

Hillert A, Koch S (2006) Berufsbezogene Psychotherapie?! Realexistierende Patienten - Therapeutische Perspektiven - Pragmatische Behandlungsansätze. In: Weber A, Hörmann G (Hrsg.) „Mensch und Neue Arbeitswelt“ Psychosoziale Gesundheit im Beruf. Stuttgart: Gentner Verlag, S. 447-458

Hillert A, Koch S, Hedlund S (2007) Stressbewältigung am Arbeitsplatz. Ein stationäres Gruppentherapieprogramm. Göttingen: Vandenhoeck \& Ruprecht

Hillert A, Marwitz M (2006) Die Burnout-Epidemie, oder: Brennt die Leistungsgesellschaft aus? Beck-Verlag München

Hillert A, Müller-Fahrnow W, Radoschewski FM (Hrsg.) (2009) Medizinisch-beruflich orientierte Rehabilitation. Deutscher Ärzteverlag: Reihe Rehabilitation, Köln

Hupfer K (2007) Suchtmittelmissbrauch am Arbeitsplatz. In: Weber A, Hörmann G (Hrsg.) „Mensch und Neue Arbeitswelt" - Psychosoziale Gesundheit im Beruf. Stuttgart: Gentner Verlag, S. 184-199

Jahnke W, Erdmann G, Kallus KW (2002) Stressverarbeitungsfragebogen mit SVF 120 und SVF 78 (3. Auflage). Göttingen: Hogrefe

Keupp H, Ahbe T, Gmür W, Höfer R, Mitzscherlich B, Kraus W, Sraus F (2008) Identitätskonstruktionen. Das Patchwork der Identitäten in der Spätmoderne. Rowohlt, Hamburg

Larisch M, Joksimovic L, von dem Knesenbeck 0, Starke D, Siegrist I (2003) Berufliche Gratifikationskrisen und depressive Symptome: Eine Querschnittsstudie bei Erwerbstätigen im mittleren Erwachsenenalter. Psychotherapie, Psychosomatik und Medizinische Psychologie 53, 223-228

Lehr D, Schmitz E, Hillert A (2008) Bewältigungsmuster und psychische Gesundheit. Eine clusteranalytische Untersuchung zu Bewältigungsmustern im Belastungskontext des Lehrerberufes. Zeitschrift für Arbeits- und Organisationspsychologie $52,3-16$

Linden M, Weidner C (2005) Arbeitsunfähigkeit bei psychischen Störungen. Nervenarzt 76, 1421-1431

Radoschewski FM (2009) Epidemiologie gesundheitsbedingter eingeschränkter Erwerbstätigkeit. In: Hillert A, Müller-Fahrnow W, Radoschewski FM (Hrsg.) Medizinisch-beruflich orientierte Rehabilitation. Deutscher Ärzteverlag: Reihe Rehabilitation, Köln, S. 14-33

Rohde A, Marneros A (Hrsg.) (2007) Geschlechtsspezifische Psychiatrie und Psychotherapie. Kohlhammer Stuttgart

Runge TR, Frey F, Gollwitzer PM, Helmreich RL, Spence JT (1981) Masculine (instrumental) and feminine (expressive) traits. A comparison between students in the United States and West Germany. Journal of Cross-Cultural Psychology 12, 142-162

Schaarschmidt U, Fischer AW (2001) Bewältigungsmuster im Beruf: Persönlichkeitsunterschiede in der Auseinandersetzung mit der Arbeitsbelastung. Göttingen: Vandenhoeck \& Ruprecht

Schaarschmidt U, Fischer AW (2003) AVEM - Arbeitsbezogenes Verhaltens- und Erlebensmuster. Frankfurt/M.: Swets \& Zeitlinger

Schaarschmidt U, Klieschke U (Hrsg.) (2007) Gerüstet für den Schulalltag. Beltz Verlag Weinheim, S. 24ff

Schulz P, Schlotz W, Wolf I, Wüst S (2002) Geschlechtsunterschiede bei stress ... Variablen: Der Einfluss der Neigung zur Besorgnis. Zeitschrift für Differentielle und Diagnostische Psychologie 23, 305-326

Sieverding M (2005) Geschlecht und Gesundheit. Enzyklopädie der Psychologie. Bd. 1 Gesundheitspsychologie, Hogrefe Göttingen, 55-70

Sieverding M, Alfermann D (1992) Geschlechtsrolle und Geschlechtsstereotype. Instrumentelles (maskulines) und expressives (feminines) Selbstkonzept: ihre Bedeutung für die Geschlechtsrollenforschung. Zeitschrift für Sozialpsychologie 6-15

Sieverding M, Weidner G, v Volkmann B (2005) Cardiovascular Reactivity in a simulated job interview: the role of gender role self-concept. Int I of Behavioral Medicine 12, 1-10

Taylor SE, Cousino Klein L, Lewis BP, Gruenewald TL, Gurung RA, Updegraff JA (2000) Biobehavioral response to stress in females: Tend-and-befriend, not fight-flight. Psychological review 107, 411-429

Toker S, Shirom A, Shapira I, Berliner S, Melamed S (2005) The association between burnout, depression, anxiety, and inflammation biomarkers: C-reactive protein and fibrinogen, in men and women. Journal of Occupational Health Psychology 10, 344-362 
2 Exkurs: Zwischen Konkurrenz, Karriere und Kollaps

Weber A, Hörmann G (Hrsg.) (2007) „Mensch und Neue Arbeitswelt“ - Psychosoziale Gesundheit im Beruf. Stuttgart: Gentner Verlag

Wietasch A-K, Kiefer M, Spitzer M (2007) Neuropsychologie. In: Rohde A, Marneros A (Hrsg.) Geschlechtsspezifische Psychiatrie und Psychotherapie. Kohlhammer Stuttgart, 305-323

Zapf D, Semmer NK (2004) Stress und Gesundheit in Organisationen. Göttingen, Hogrefe (Organisationspsychologie - Grundlagen und Personalpsychologie; Enzyklopädie der Psychologie Serie III, Bd. 3 